\title{
Progredienter inspiratorischer Stridor nach Verbrennungstrauma des Larynx
}

\section{Einleitung}

Durch Verbrennungstraumata des Larynx können dauerhafte Einschränkungen der Funktionen des Kehlkopfes resultieren. Dabei wird unmittelbar auftretenden glottischen und supraglottischen Läsionen im Vergleich zu Trachea und unteren Atemwegen initial häufig weniger Beachtung geschenkt. Eine persistierende Dysphonie stellt eine häufige Spätfolge laryngealer Verbrennungen dar.

\section{Fallbericht}

Wir berichten über eine 19-jährige Patientin, die sich aufgrund einer Dysphonie und geringgradiger Belastungsdyspnoe im Oktober 2008 in unserer Abteilung vorstellte. Zwei Monate zuvor hatte sie sich in der Freizeit beim Feuerschlucken im Bereich der oberen Atemwege eine Verbrennung 1. Grades zugezogen, die auswärtig intensivmedizinisch behandelt worden war.

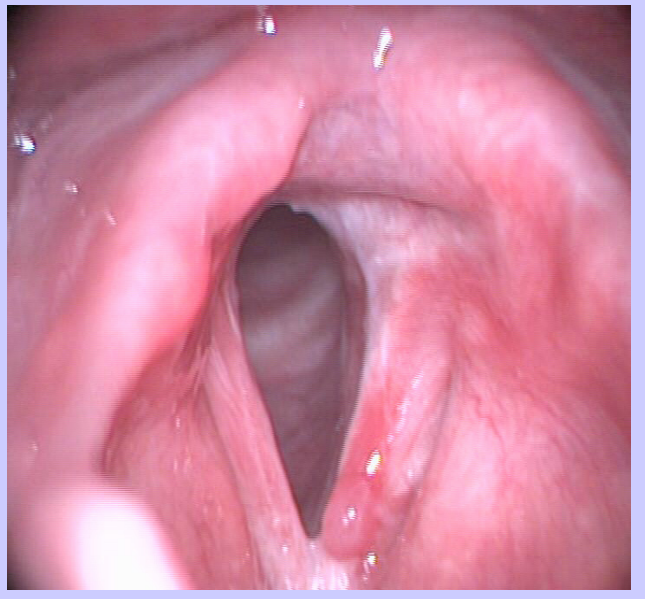

Abb. 1

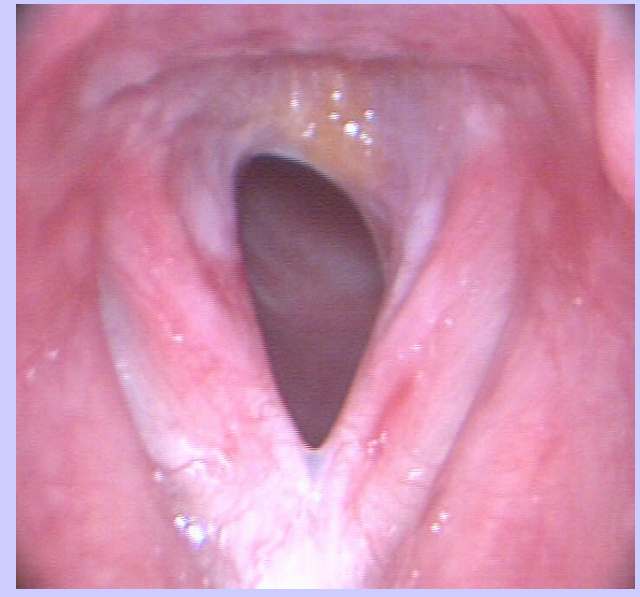

Abb. 2

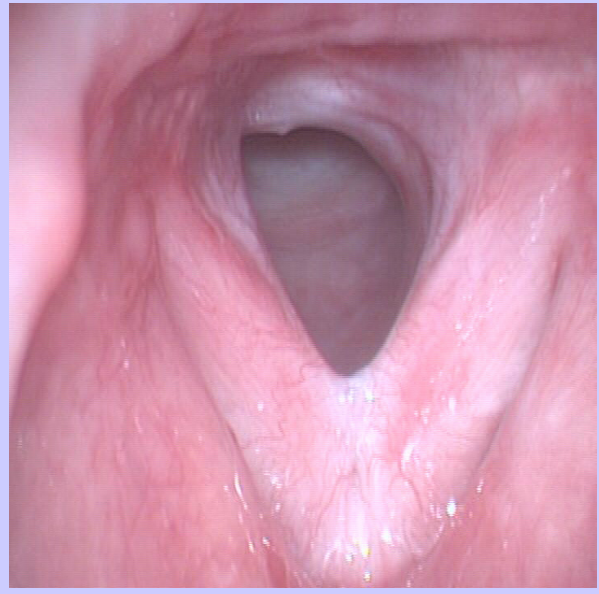

Abb. 3

\section{Befund}

Bei der lupenlaryngoskopischen Untersuchung imponierte eine granulomatöse Schleimhautläsion im Bereich des vorderen Drittels der linken Stimmlippe (Abb 1). Durch logopädische Therapie konnte eine Linderung der Stimm- und Atembeschwerden erreicht werden.

\section{Verlauf}

Die Kontrolluntersuchung im Februar 2009 zeigte Vernarbungen der Stimmlippenschleimhaut und Synechien im Bereich der vorderen und der hinteren Kommissur (Abb. 2). Wir empfahlen eine Inhalationstherapie mit einer Natriumchlorid-Lösung sowie die Fortsetzung der logopädischen Stimmtherapie.

Vier Monate später trat ein inspiratorisches Atemgeräusch auf. Im Bereich der hinteren Kommissur hatte sich ein Narbensegel gebildet, welches zu einer subglottischen Stenosierung geführt hatte und eine laserchirurgische Intervention erforderlich machte (Abb 3). Im postoperativen Verlauf kam es zu einer deutlichen Rückbildung der Vernarbung und zu einer Besserung der Stimme.

\section{Schlussfolgerung}

Verbrennungsverletzungen des Kehlkopfes sind selten. Initial steht das Schleimhautödem im Vordergrund, das sich durch Cortison und Inhalationsbehandlungen schnell zurückbildet. Im weiteren Verlauf kann es jedoch zu Vernarbungen kommen, die häufig - in Abhängigkeit der Symptomatik - eine operative Therapie verlangen. Hierzu stehen laserchirurgische Verfahren zur Verfügung. In manchen Fällen kann die Heilung zusätzlich durch Anwendung von Platzhaltern unterstützt werden.

\section{Literatur}

Valdez TA, Desai U, Ruhl CM, Nigri PT. Early Laryngeal Inhalation Injury and its Correlation with late sequelae. Laryngoscope 2006;116:283-7 Casper JK, Clark WR, Kelley RT, Colton RH.Laryngeal and Phonatory Status After Burn/ Inhalation Injury: A Long Term Follow-Up Study. J Burn Care Rehabil 2002; 23:235-243. 